\title{
Picosecond infrared activation of methanol in acid zeolites
}

\author{
Mischa Bonn ${ }^{\mathrm{a}, \mathrm{l}, \mathrm{b}}$, Rutger A. van Santen ${ }^{\mathrm{b}}$, Johannes A. Lercher ${ }^{\mathrm{c}}$, Aart W. Kleyn ${ }^{\mathrm{a}}$, \\ Huib J. Bakker ${ }^{\text {a }}$ \\ "FOM-Institute for Atomic and Molecular Physics, Kruislaan 407, 1098 SJ Amsterdam, The Netherlands \\ ${ }^{h}$ Schuit Institute of Catalysis, Eindhoven University of Technology, P.O. Box 513,5600 MB Eindhoven. The Netherlands \\ "Faculty of Chemical Technology, Twente Unicersity of Technology, P.O. Box 217, 7500 AE Twente, The Netherlands
}

Received 1 July 1997; in final form 1 September 1997

\begin{abstract}
Highly porous, crystalline zeolite catalysts are used industrially to catalyze the conversion of methanol to gasoline. We have performed a picosecond spectroscopic study providing insights into both the structure and the dynamics of methanol adsorbed to acid zeolites. We reveal the adsorption structure of methanol at the catalytically reactive site and show that the reaction of this complex in the zeolite to the first intermediate in the methanol-to-gasoline reaction sequence is initiated by picosecond infrared excitation. The picosecond dynamics of this activation process can be followed in real-time, and reflects the potential energy surface relevant to the reaction. (C) 1997 Elsevier Science B.V.
\end{abstract}

\section{Introduction}

With the advent of ultra-short laser pulses, it has become possible to follow reaction dynamics 'realtime': ultra-short laser pulses can be used to initiate a chemical reaction and subsequently probe (and even control) its time-evolution [1]. Although these experiments have indisputably enriched our understanding of chemical reaction dynamics, they have been mostly limited to (gas-phase) model systems. Here we demonstrate that picosecond infrared pulses can be used to investigate the structure and dynamics of the industrially relevant system of methanol adsorbed to the active sites in acid zeolite catalysts. Zeolites are used to convert methanol to gasoline. Although this process is a potentially important alter-

\footnotetext{
${ }^{\prime}$ Present address: Fritz-Haber Institut der Max-Planck-GeselIschaft. Faradayweg 4-6, D-14195 Berlin, Germany.
}

native source of (environmentally friendly) fuels, a fundamental understanding of the structure and dynamics of methanol adsorbed to zeolites is still lacking. In this time-resolved spectroscopic study, mechanistic insights are obtained into the dynamics and the molecular basis of the elementary reaction steps in zeolites. Reaction intermediates can be created and probed real-time.

Acid zeolites are crystalline alumino-silicates with a high internal surface area $\left(\sim 1000 \mathrm{~m}^{2} / \mathrm{g}\right)$, containing acidic hydroxyl $(\mathrm{O}-\mathrm{H})$ groups located in nanometer micropores [2]. The catalytic activity lies in the fact that these hydroxyls can 'lend' their proton to a reactant adsorbed in the zeolite structure [2]. Since these solid acids are able to catalyze the transformation of methanol to light olefins (e.g. hydrocarbon fuels in the methanol-to-gasoline process [3]), it is important to elucidate the methanol adsorption structures, its activation at the zeolite acid site 
and subsequent reaction mechanisms [4,5]. In particular, knowledge of the dynamics of this catalytic system on a molecular scale is essential for a fundamental understanding of the methanol-to-gasoline conversion process. We investigate methanol adsorbed to the acidic form of zeolite Y [6], a generic catalytic system, with both conventional and picosecond infrared spectroscopy and with thermogravimetry, a technique which provides the heat of adsorption as a function of methanol loading.

\section{Experimental}

The zeolite used for the experiments described in this Letter was $\mathrm{Y}$ zeolite with a silicon/aluminum ratio of $\mathrm{Si} / \mathrm{Al}=2.7$ and a proton content of $\mathrm{H} /(\mathrm{Si}+\mathrm{Al})=0.22$ (corresponding to $82 \%$ protons and $18 \% \mathrm{Na}^{+}$ions). The presence of the sodium ions does not affect the outcome of the experiments, because the adsorption of methanol occurs preferentially at the acid site. Zeolite $\mathrm{Y}$ was chosen for its relatively high density of acid sites, required for the observation of significant transmission changes in the time-resolved experiments. Absorption spectra were recorded using a Perkin-Elmer 881 double beam IR-spectrometer. The absorption and time-resolved experiments were performed in transmission through a $\sim 70 \mu \mathrm{m}$ thick, pressed self-supporting disc of zeolite crystallites.

For the time-resolved experiments picosecond ( 20 ps) tunable infrared $\left(2500-4000 \mathrm{~cm}^{-1}\right)$ pulses of $\sim 150 \mu \mathrm{J}$ energy (bandwidth $\sim 20 \mathrm{~cm}^{-1}$ ) are generated by downconversion of $9400 \mathrm{~cm}^{-1}(1064 \mathrm{~nm})$ pulses from a $\mathrm{Nd}$ :YAG laser. The changes in the absorption spectrum due to excitation with an IR pulse are monitored by weak $(3 \mu \mathrm{J})$, independently tunable, infrared pulses. The time delay between the excitation and probe pulses can be variably delayed, by increasing the pathlength of the probe $(1 \mathrm{~mm} \propto$ $3.33 \mathrm{ps})$. An extensive description of the apparatus can be found in Ref. [7].

\section{Results and discussion}

The absorption spectrum of proton-exchanged $\mathrm{Y}$ zeolite, $\mathrm{H}_{0.22} \mathrm{Y}$, depicted in Fig. 1, shows two peaks

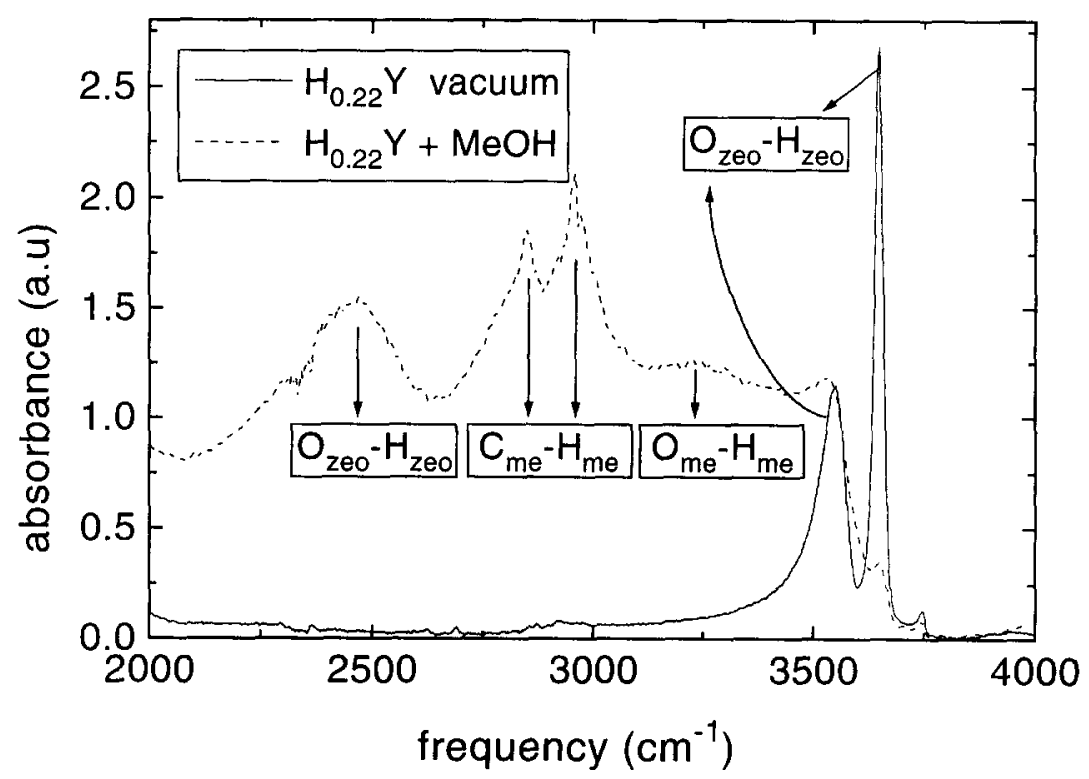

Fig. 1. Absorption spectra of $\mathrm{H}_{0.22} \mathrm{Y}(\mathrm{Si} / \mathrm{Al}=0.27$ ), in vacuum (solid line) and with adsorbed methanol (dashed line, final pressure $P_{\mathrm{MeOH}}=3 \times 10^{-2} \mathrm{mbar}$ ). The assignment of the different absorption peaks is indicated in the graph. The presence of the strongly hydrogen-bonded feature of the zeolite $\mathrm{O}_{\mathrm{zeo}}-\mathrm{H}_{\text {zeo }}$ stretching vibration at $2500 \mathrm{~cm}^{-1}$, indicates that no proton transfer takes place between the zeolite hydroxyl and the methanol $[9,10]$. 
at 3550 and $3645 \mathrm{~cm}^{-1}$, associated with the $\mathrm{O}\left\{\leftarrow_{\text {zeo }}\right.$ $-\mathrm{H}\left\{\rightarrow{ }_{\text {zeo }}\right.$ stretching vibration of the acidic zeolite hydroxyls sticking into the small and large cages of the zeolite [8]. The same figure demonstrates that, upon adsorption of methanol (at a steady state methanol pressure $P_{\mathrm{MeOH}}=3 \times 10^{-2} \mathrm{mbar}$ ), the absorption at these frequencies decreases because the peaks are strongly redshifted due to the strong hydrogen-bonding interaction between the zeolite hydroxyls and the methanol. This results in, among other spectral features, the appearance of a broad peak at around $2500 \mathrm{~cm}^{-1}[9,10]$. Sharp peaks appear at around 2800 and $2900 \mathrm{~cm}^{-1}$ which are associated with the $\mathrm{C}-\mathrm{H}$ stretching vibration of the adsorbed methanol [11]. For methanol partial pressures above $\sim 1 \times 10^{-2}$ mbar, a broad peak at around $3250 \mathrm{~cm}^{-1}$ is clearly observed, which is due to the $\mathrm{O}_{\mathrm{me}}-\mathrm{H}_{\mathrm{me}}$ stretching vibration of the methanol hydroxyl adsorbed to the acid site [11,12]. The frequency of the $\mathrm{O}_{\mathrm{me}}-\mathrm{H}_{\mathrm{me}}$ stretching vibration (3250 $\mathrm{cm}^{-1}$ ) is low compared to the frequency of gas-phase methanol $\left(3681 \mathrm{~cm}^{-1}\right)$. This is caused by a $\mathrm{CH}_{3} \mathrm{O}-$ $\mathrm{H} \cdots \mathrm{O}$ hydrogen bond between the methanol hydroxyl and an oxygen atom. An important question is whether this oxygen atom belongs to the zeolite lattice or to other methanol molecules, which depends on the exact microscopic adsorption configuration. This structure might be a single methanol molecule, a methanol dimer, or a larger cluster of methanol adsorbed to the acid sites.

In order to investigate the adsorption structure, we selectively excite the $\mathrm{O}_{m e}-\mathrm{H}_{\mathrm{mc}}$ stretching vibration of methanol adsorbed to the acid site at $3250 \mathrm{~cm}^{-1}$ with a powerful $(\sim 150 \mu \mathrm{J})$ picosecond $(20 \mathrm{ps})$ infrared pulse, tuned to this frequency. The changes in the absorption spectrum (and the time evolution) due to the excitation, are monitored by a second weak $(3 \mu \mathrm{J})$, independently tunable (probe) pulse, with a variable time delay. Fig. 2. reveals that, 270 ps after excitation the absorption decreases at the excitation frequency $\left(\tilde{\nu}=3250 \mathrm{~cm}^{-1}\right)$, whereas at around $\tilde{\nu}=3580 \mathrm{~cm}^{-1}$ an increased absorption is

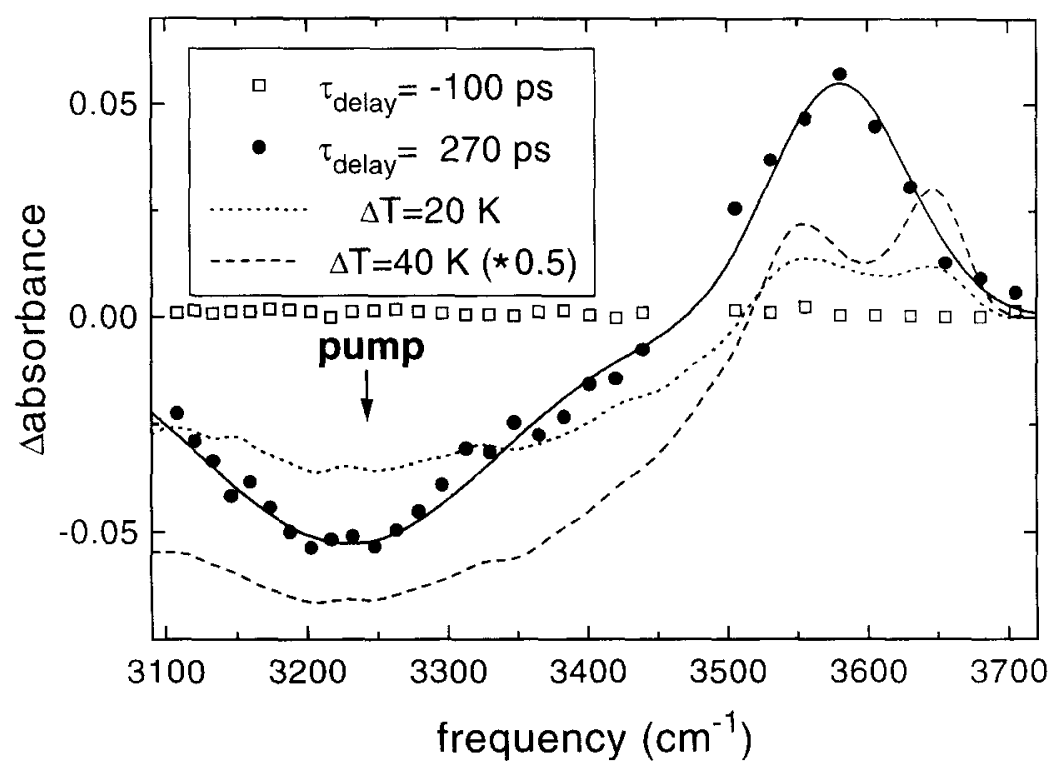

Fig. 2. Absorption changes after excitation at $3250 \mathrm{~cm}^{-1}$ of the methanol:HY system corresponding to the dashed absorption spectrum in Fig. 1. For negative delay between excitation and probe pulse, $\left(\square \tau_{\text {delay }}=-100 \mathrm{ps}\right.$ ), the probe hits the sample before the excitation (no absorption changes $)$. For longer delays $\left(-\tau_{\text {delay }}=270 \mathrm{ps}\right)$ the absorption decreases around the excitation frequency $\left(3250 \mathrm{~cm}^{-1}\right)$, while simultaneously an induced absorption is observed around $3580 \mathrm{~cm}^{-1}$. The solid line through the data is a calculation with a disappearing

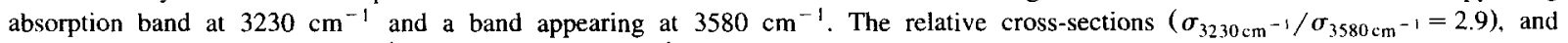
linewidths $\left(\Gamma_{3230 \mathrm{~cm}^{-1}}=250 \mathrm{~cm}^{-1}, \Gamma_{3580 \mathrm{~cm}^{-1}}=100 \mathrm{~cm}^{-1}\right)$, support the notion of a weakened hydrogen bond, since both the cross-section and the linewidth are known to decrease with decreasing hydrogen-bond strength [13]. Dashed and dotted lines show the effect of simple temperature increases, leading to the thermal desorption of methanol, which clearly cannot describe the data (see footnote). 
observed: The excitation causes a frequency shift of the $\mathrm{O}_{\mathrm{me}}-\mathrm{H}_{\mathrm{me}}$ stretching vibration of adsorbed methanol from 3250 to $3580 \mathrm{~cm}^{-1}$. Since there exists a strong correlation between the hydrogen-bond strength and the vibrational frequency [13], the higher frequency of the methanol $\mathrm{O}_{\mathrm{me}}-\mathrm{H}_{\mathrm{me}}$ indicates that due to the excitation, the $\mathrm{CH}_{3} \mathrm{O}-\mathrm{H} \cdots \mathrm{O}$ hydrogen bond of the methanol hydroxyl group is dissociated. The observed absorption changes cannot be explained by a simple temperature increase. The effect of typical temperature increases [14] on the absorption spectrum are shown as dotted $(\Delta T=20 \mathrm{~K})$ and dashed lines $(\Delta T=40 \mathrm{~K})$ in Fig. 2. With increasing temperature, thermal desorption of some methanol occurs, giving rise to increased absorption at the $\mathrm{O}_{\text {zeo }}-\mathrm{H}_{\text {zeo }}$ frequencies ( 3550 and $3645 \mathrm{~cm}^{-1}$ ), clearly different from the data ${ }^{2}$. The dissociation of the $\mathrm{CH}_{3} \mathrm{O}-\mathrm{H} \cdots \mathrm{O}$ hydrogen bond can be understood by noting that because of the strong coupling between the $\mathrm{O}-\mathrm{H}$ stretch vibration and the $\mathrm{CH}_{3} \mathrm{O}$ $\mathrm{H} \cdots \mathrm{O}$ hydrogen bond, relaxation of the energy in the excited $\mathrm{O}-\mathrm{H}$ groups leads to dissociation of this hydrogen bond. This has been demonstrated for excited $\mathrm{O}-\mathrm{H}$ groups of methanol [15] and ethanol [16] clusters in solution, adsorbed to NaY-zeolite [15], in the gas phase [17] and in a nitrogen-matrix [18].

The picosecond time-resolved transmission changes, which reflect the dynamics of the hydrogen-bond dissociation of methanol at the acid site, are shown in Fig. 3 for low $\left(P_{\mathrm{MeOH}}=3 \times 10^{-2}\right.$ mbar $)$ and high $\left(P_{\mathrm{MeOH}}=20\right.$ mbar $)$ methanol pressures. In these experiments, the delay between the excitation ('pump', at $3250 \mathrm{~cm}^{-1}$ ) and probe pulses (at 3250 and $3580 \mathrm{~cm}^{-1}$ ) is scanned. Intriguingly, there is a significant and reproducible difference in the dynamics of the $\mathrm{H}$-bond dissociation process: at high pressures, the dynamics of the methanolmethanol hydrogen-bond dissociation is characteris-

\footnotetext{
${ }^{2}$ After thermal equilibration of the absorbed infrared energy, a temperature gradient will be present over the sample, due to the relatively high absorbance (more light will be absorbed at the beginning of the sample compared to the end). The measured transient of Fig. 2 cannot be explained by averaging over different temperature increases, since the observed ratio between transmission increase and transmission decrease is much larger than for any temperature increase.
}

tic of normal hydrogen-bonded systems, viz. fast $[15,16]$, probably sub-picosecond dissociation dynamics. Due to these fast dynamics (much faster than our experimental time-resolution), the transmission changes linearly with the amount of deposited energy, following the integral of the excitation pulse envelope. In contrast, at low pressure, the behaviour is anomalous: the transmission changes also set in at around delay zero, but for longer delay times the response is substantially slower. The differences between the observed dynamics suggests that different adsorption complexes are excited in going from $P_{\mathrm{MeOH}}=3 \times 10^{-2}$ mbar to higher pressures.

Correspondingly, thermogravimetric experiments reveal a sharp drop of $\sim 30 \mathrm{~kJ} / \mathrm{mol}$ in the adsorption energy at $P_{\mathrm{MeOH}}=3 \times 10^{-2} \mathrm{mbar}$. The same experiments reveal that at this pressure, the average occupation of Brønsted sites by the methanol molecules (in both the small and large cages) is $1.3 \pm 0.1$, whereas from the remaining intensity at 3550 and $3645 \mathrm{~cm}^{-1}$ in the absorption spectrum, it is clear that some of the zeolite acidic sites are still unoccupied. In combination with the infrared spectrum and the time-resolved data, these results strongly indicate the following pressure-dependent adsorption structures: although at sufficiently low pressures $\left(P_{\mathrm{MeOH}} \ll 1 \times 10^{-2}\right.$ mbar $)$, monomeric adsorption will occur, at pressures of around $P_{\mathrm{MeOH}}=3 \times 10^{-2}$ mbar, the preferential adsorption structure is two methanol molecules at one site, even though not all the acid sites are occupied. At even higher loading larger clusters will be formed. This explains both the drop in the adsorption energy when the average occupation number exceeds one methanol molecule per acid site and the differences between the dynamics below and above $P_{\mathrm{MeOH}}=3 \times 10^{-2}$ mbar. The adsorption structure of the methanol dimer, present at pressures below $3 \times 10^{-2}$ mbar and the frequencies of the $\mathrm{O}_{\mathrm{me}}-\mathrm{H}_{\mathrm{me}}$ groups are depicted in Fig. 4A. The $3250 \mathrm{~cm}^{-1}$ absorption emerging upon formation of the dimer must be associated with the $\mathrm{O}_{\mathrm{me}}-\mathrm{H}_{\mathrm{me}}$ vibration of the methanol adsorbed to the acid site, hydrogen-bonded to the second methanol molecule. The $\mathrm{O}_{\mathrm{me}}-\mathrm{H}_{\mathrm{me}}$ group of the second methanol molecule interacts weakly with oxygen atoms in the zeolite lattice and its vibrational frequency will resemble that of monomerically adsorbed methanol, absorbing at around $3500 \mathrm{~cm}^{-1}$ [12] (we cannot 

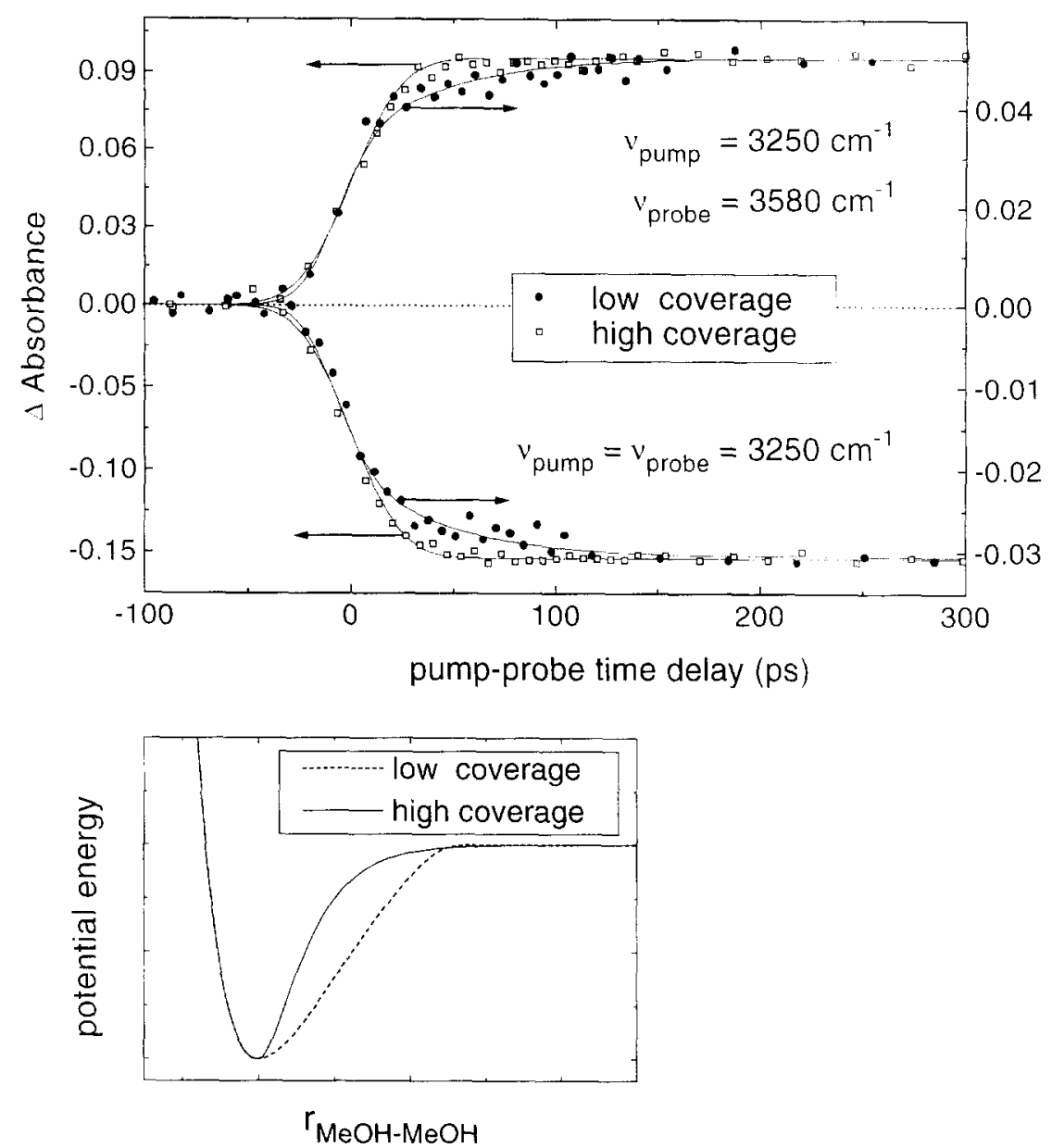

Fig. 3. Upper panel: Time-resolved measurement of the spectral changes after excitation at $3250 \mathrm{~cm}^{-1}$ of methanol:HY, at 3250 (lower traces) and $3580 \mathrm{~cm}^{-1}$ (upper traces), at low coverage (O) compared to the large, unreactive clusters at higher coverage ( $\square$ ). Note that the dynamic response is markedly different for the reactive methanol dimer at low coverage (O) compared to the large. unreactive clusters at higher coverage ( $\square$ ). The absorption changes persist up to the largest experimental delay time of $1.5 \mathrm{~ns}$. Lines are guides to the cye. Lower panel: schematic intermolecular (methanol-methanol) potentials for the reactive methanol dimer at low coverage (dashed line, anharmonic) compared to the large, unreactive clusters at higher coverage (solid line).

observe this feature directly due to overlap with absorption of the zeolite $\mathrm{O}-\mathrm{H}$ ).

Knowledge of the exact adsorption structure and whether or not clusters of methanol are formed, is important since the adsorption structure determines the reactivity: The first step in the catalytic conversion of methanol is the association reaction of two methanol molecules to give water and dimethyl-ether (DME, $\mathrm{CH}_{3}-\mathrm{O}-\mathrm{CH}_{3}$ ). If a single methanol is adsorbed, initially dehydration of a single adsorbed methanol takes place, resulting in a methoxy group at the site. A second methanol can subsequently react with this methoxy group to form DME. In contrast to this highly activated, consecutive process, a second mechanism is formed by the coadsorption of two or more methanol molecules that react simultaneously at the zeolite hydroxyl, to form DME directly. Recent density functional theory calculations suggest that the energy barrier for DME formation is substantially lower for the second process [19]. The 

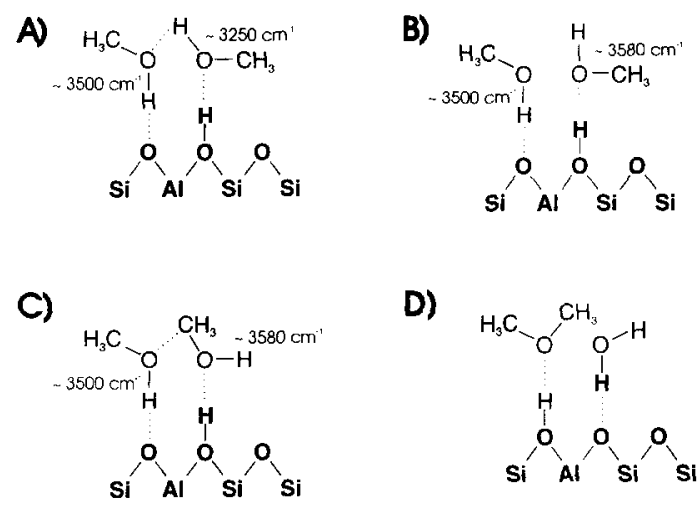

Fig. 4. Schematic representations of two methanol molecules (depicted by normal letters) interacting with a zeolite acid site (bold letters). (A) In equilibrium, (B) with dissociated intermolecular hydrogen bond, (C) rotated, so the two methyl groups are sufficiently near to react and (D) after the reaction, by which di-methyl ether is formed. The frequencies of the methanol $\mathrm{O}_{\mathrm{me}}-$ $\mathbf{H}_{\mathrm{me}}$ stretching vibrations are indicated in the graph.

dimer adsorption structure which we identify, is therefore the specific starting point for the formation of gasoline from methanol.

Before the reaction of the two methanol molecules co-adsorbed at the acid site can occur, the hydrogen bond between the two methanol molecules has to be dissociated (see Fig. 4B), so that the methanol adsorbed to the acid site can rotate (see Fig. 4C), thereby bringing the methyl groups together to form DME [19] (see Fig. 4D). Hence, the dissociation of the hydrogen bond between the two methanol molecules $(4 \mathrm{~A} \rightarrow 4 \mathrm{~B})$, induced by the infrared pulse, is the first step in the catalytic reaction of the methanol dimer at the acid site: the new absorption observed at $3580 \mathrm{~cm}^{-1}$ after excitation of the dimer at $3250 \mathrm{~cm}^{-1}$ is due to the first reaction intermediate (Fig. 4B) in the methanol-to-gasoline sequence; the reaction is 'switched on' by the infrared excitation. It should be stressed that we do not observe the formation of DME directly. The reaction of the two methanol molecules is initiated by the infrared excitation in the sense that dissociation of the hydrogen bond is a prerequisite for the reaction and this is brought about by the infrared pulse.

The hydrogen-bond dissociation dynamics, depicted in Fig. 3, are clearly different for low and high methanol pressures. This difference can be tentatively understood by considering the catalytic ac- tivity of the system at low pressures. For the dimer at low pressure, the hydrogen-bond dissociation takes place at the zeolite catalytic site. It is typical of catalysts that energy barriers are lowered, so that potential energy surfaces will be deformed. Since, for low loading, the hydrogen-bond dissociation is the first step in the catalytic reaction for the methanol dimer, the methanol-methanol interaction potential will be strongly affected by the zeolite catalyst. In contrast, at higher loading (viz. pressure), the methanol molecules experience a much less reactive environment, since they are predominantly hydrogen-bonded to other methanol molecules. Therefore excitation of the large cluster (at high loading) leads mainly to H-bond dissociation of methanol hydroxyls within the cluster, far from the reactive site. At high pressures, the potential will therefore remain largely unaffected for methanol molecules far from the catalytic site and resemble that of e.g. liquid methanol. This is schematically depicted at the bottom of Fig. 3: The shape of the methanol-methanol interaction potential is different for low and high loading. These tentative potentials illustrate that for low loading, the potential will be more anharmonic when compared to the potential for the high-pressure situation. Although additional experiments will be required to obtain the precise shape of the potentials, it is clear that there must be a difference between the intermolecular potentials for low and high loading to account for the difference in dynamics that is observed. In both cases the hydrogen bond is dissociated due to the infrared excitation; the infrared energy is used to advance upwards in the potential well. It is important to note that the dissociation is not a one-photon process in the sense that the energy of a single photon is inserted into a single hydrogen bond. Rather, the dissociation is due to a redistribution of the excess vibrational energy over a limited number of (vibrational) degrees of freedom (it is clear from Fig. 2 that complete thermalization does not occur). This is confirmed by experiments in which the $\mathrm{C}-\mathrm{H}$ stretch of methanol and the $\mathrm{O}_{z e o}-\mathrm{H}_{\text {zeo }}$ stretch vibrations were excited. Due to the increased anharmonicity for the reactive and strongly perturbed dimer, the mean methanol-methanol distance depends non-linearly on the amount of energy put into the system: energy which has been preceded by a certain amount of energy causes a greater change in 
the distance than the same amount of energy alone. This non-linearity is mirrored in the time-resolved data. The change in the frequency of the $\mathrm{O}_{\mathrm{me}}-\mathrm{H}_{\mathrm{me}}$ stretching vibration directly reflects the change in the methanol-methanol distance [13]. As a result, the observed transmission change (Fig. 3) is extended, protracted for the dimer. These results demonstrate the possibility of using non-linear spectroscopy to induce and to follow real-time the activation of catalytically active, reactive complexes. Our results call for a theoretical or computational approach for this system that includes the dynamics of the reaction path.

\section{Conclusion}

Summarizing, we demonstrate that picosecond infrared spectroscopy is a powerful tool in identifying the adsorption structure of methanol adsorbed to the zeolite acid site. In addition, we show that this reactive complex can be activated by means of infrared excitation. The real-time dynamics of this activation process reflects the potential between the two methanol molecules at the catalytically active site that is relevant to the reaction.

\section{Acknowledgements}

We kindly acknowledge S.R. Blaszkowski for many helpful discussions. This work is part of a collaborative research program of the Stichting Fundamenteel Onderzoek van de Materie (Foundation for Fundamental Research on Matter) with financial support from the Nederlandse Organisatie voor Wetenschappelijk Onderzoek (Netherlands Organiza- tion for the Advancement of Research), and the Schuit Institute of Catalysis at the Eindhoven University of Technology.

\section{References}

[1] E.D. Potter, J.L. Herek, S. Pedersen. Q. Liu, A.H. Zewail, Nature 533 (1992) 66.

[2] J.M. Thomas, Scient. Am. 266 (IV) (1992) 82.

[3] C.D. Chang, Hydrocarbons from methanol, Marcel Dekker, New York. 1983.

[4] R. Shah, M.C. Payne, J.D. Gale, Science 27I (1996) 1395.

[5] G. Mirth, J.A. Lercher, M.W. Anderson. J. Klinowski. J. Chem. Soc. Faraday Trans. 86 (1990) 3039.

[6] W.M. Meier. D.J. Olson, Ch. Baerlocher. Atlas of zeolite structure types, 4th rev. ed.. Elsevier, London, 1996.

[7] M.J.P. Brugmans, H.J. Bakker. A. Lagendijk, J. Chem. Phys. 104 (1996) 64.

[8] M. Czjzek. H. Jobic. H. Fitch. T. Vogt. J. Phys. Chem. 96 (1992) 1535.

[9] A.G. Pelmenshikov, J.H.M.C. van Wolput, J. Jänchen. R.A. van Santen, J. Phys. Chem. 99 (1995) 3612.

[10] F. Haase, J. Sauer, J. Am. Chem. Soc. 117 (1995) 3780.

[11] G. Mirth, A. Kogelbauer, J.A. Lercher, in: Proc. 9th Int Zeol. Conf. Montreal (1992), R. von Ballmoos et al.. Eds. (Butterworth-Heinemann, USA. 1993) pp. 251-258.

[12] A. Zecchina, S. Bordiga, G. Spoto, D. Scarano, G. Spano. F. Geobaldi. J. Chem. Soc. Faraday Trans. 92 (1996) 4863.

[13] D. Hadži, S. Bratos, in: The Hydrogen Bond, Vol. 2. P. Schuster et al., Eds. (North-Holland. Amsterdam. 1976) pp. $565-613$.

[14] M. Bonn, M.J.P. Brugmans, A.W. Kleyn. R.A. van Santen, J. Chem. Phys. 102 (1995) 2181.

[15] M. Bonn. H.J. Bakker, A.W. Kleyn. R.A. van Santen, J. Phys. Chem. 100 (1996) 15301.

[16] H. Graener. T.Q. Ye, A. Laubereau. J. Chem. Phys. 90 (1989) 3413.

[17] F. Huisken, A. Kulcke. C. Laush. J.M. Lisy, J. Chem. Phys. 95 (1991) 3924.

[18] S. Coussan, N. Bakkas, A. Loutellier. J.P. Perchard, S. Racine, Chem. Phys. Lett. 217 (1994) 123.

[19] S.R. Blaszkowski, R.A. van Santen, J. Am. Chem. Soc. 118 (1996) 5152, and references therein. 